\title{
Association Study of the Complement Component C4 Gene in Tardive Dyskinesia
}

\author{
Clement C. Zai ${ }^{1,2,3,4 *}$, Arun K. Tiwari ${ }^{1,2}$, Gwyneth C. Zai ${ }^{1,2,3}$, Natalie Freeman', \\ Jennie G. Pouget ${ }^{1,2}$, James Greco ${ }^{1}$, Maria Tampakeras ${ }^{1}$, Sajid A. Shaikh', \\ Deanna Herbert ${ }^{1}$, Heather Emmerson ${ }^{1}$, Sheraz Y. Cheema ${ }^{1}$, Nicole Braganza ${ }^{1}$, \\ Daniel J. Müller ${ }^{1,2,3}$, Aristotle N. Voineskos ${ }^{1,2,3}$, Gary Remington ${ }^{1,2,3}$ \\ and James $L$. Kennedy $y^{1,2,3 *}$
}

${ }^{1}$ Tanenbaum Centre for Pharmacogenetics, Campbell Family Mental Health Research Institute, Centre for Addiction and Mental Health, Toronto, ON, Canada, ${ }^{2}$ Department of Psychiatry, University of Toronto, Toronto, ON, Canada, ${ }^{3}$ Institute of Medical Science, University of Toronto, Toronto, ON, Canada, ${ }^{4}$ Laboratory Medicine and Pathobiology, University of Toronto, Toronto, ON, Canada

Tardive dyskinesia (TD) is a movement disorder that may develop in schizophrenia patients being treated long-term with antipsychotic medication. TD interferes with voluntary movements and leads to stigma, and can be associated with treatment nonadherence. The etiology of TD is unclear, but it appears to have a genetic component. There is emerging evidence of immune dysregulation in TD. In the current study, we set out to investigate the complex schizophrenia-associated complement component 4 (C4) gene for possible association with TD occurrence and TD severity as assessed by the Abnormal Involuntary Movement Scale (AIMS) in a sample of 129 schizophrenia patients of European ancestry. We have genotyped the copy numbers of long and short forms of C4A and C4B gene variants in 129 European ancestry patients with schizophrenia or schizoaffective disorder. We did not find predicted C4A or C4B expression to be nominally associated with TD risk or severity. However, we found the number of copies of $C 4 \mathrm{BL}$ to be nominally associated with TD severity $(p=0.020)$.

Keywords: pharmacogenetics, tardive dyskinesia (TD), complement component C4, schizophrenia, antipsychotic

\section{INTRODUCTION}

Schizophrenia is a chronic and severe psychiatric disorder characterized by, amongst other symptoms, hallucinations and delusions. Its etiology is complex and remains unclear, but immunerelated mechanisms have been postulated (Khandaker and Dantzer, 2016; van Kesteren et al., 2017). In 2009, two genome-wide association studies implicated the major histocompatibility complex (MHC) region in schizophrenia (Shi et al., 2009; Stefansson et al., 2009). Symptoms of schizophrenia are treated with antipsychotics, which, as a medication class, is associated with risk of developing tardive dyskinesia (TD). The etiology of TD remains unclear (Lee and Kang, 2011; Zai et al., 2018a; Zai et al., 2018b), but a genetic component has been supported by family studies (Weinhold et al., 1981; Yassa and Ananth, 1981; Müller et al., 2001). 
The immune system has not been extensively studied in TD. One study reported serum interleukin-2 receptor (IL2R) levels to be increased in antipsychotic-naive or typical antipsychoticmedicated schizophrenia patients with TD versus those without (Rapaport and Lohr, 1994), while another study reported IL2 levels to be decreased in chronic-medicated schizophrenia patients with TD compared to those without (Liu et al., 2012). IL2 serum levels have also been correlated positively to TD severity as measured by the Abnormal Involuntary Movement Scale (AIMS) (An et al., 2015). In yet another such study, IL6 levels were found decreased in schizophrenia patients with TD compared to those without (An et al., 2015). Along similar lines, marginally decreased serum TNF-alpha levels were found in medicated chronic schizophrenia patients with TD versus those without (Tian et al., 2014). The TNF marker -308A/G (rs1800629) has been found to be associated with TD severity (Wang et al., 2012), whereas the IL10 markers rs1800872 and rs72393728 were not significantly associated with TD in another study (Sun et al., 2013).

The complement component 4 (C4) mediates immune function within the complement system, which is involved in the body's defense against foreign pathogens. The system consists of the classical, lectin, and alternative pathways that converge on the cleavage of $\mathrm{C} 3$ into its active fragments $\mathrm{C} 3 \mathrm{a}$ and $\mathrm{C} 3 \mathrm{~b}$, resulting in phagocytosis, inflammation, and cell lysis by formation of the membrane attack complex (reviewed in Woo et al., 2019 In Huntington's disease patients who often manifest dyskinesia reminiscent of $\mathrm{TD}$, complement components of the classical immune pathway, including $\mathrm{C} 4$, were found in their striatum, but not so in control brains (Singhrao et al., 1999). Increased levels of complement pathway components including $\mathrm{C} 4$ have been reported in serum and cerebrospinal fluids of neurodegenerative diseases such as amyotrophic lateral sclerosis (Bahia El Idrissi et al., 2016). Furthermore, $C 4$ deficiency has been shown to protect against post-injury motor deficits and brain tissue damage in the controlled cortical impact mouse model of traumatic brain injury (You et al., 2007). Of note, while the $C 4$ gene has been implicated in schizophrenia (Sekar et al., 2016), it has not been investigated in TD. The C4 gene (localized to the MHC class III region on 6p) has a complex structure. An individual may have different copy numbers of two possible isotypes of $C 4, C 4 \mathrm{~A}$ and $\mathrm{C} 4 \mathrm{~B}$, which have different molecular targets (Sekar et al., 2016). C4A and C4B also exists as either a short (S) form or long (L) form with the insertion of a human endogenous retrovirus in intron 9 (Wu et al., 2007), resulting in four potential compound structural forms of C4: C4AL, C4AS, C4BL, and C4BS. The insertion of the human endogenous retrovirus has been shown to downregulate the expression of the parent C4 gene (Yang et al., 2003; Mack et al., 2004).

\section{METHODS}

\section{Subjects}

For the current study, we included 87 participants for which the sample characteristics have been described previously (GR; Zai et al., 2007; Zai et al., 2013). Briefly, participants were enrolled from the Center for Addiction and Mental Health in Toronto, Ontario (Dr. G Remington), and were of European ancestry. Participants were diagnosed with schizophrenia or schizoaffective disorder based on DSM-III-R or DSM-IV criteria (APA, 2000 \#3). These chronic patients had been exposed to typical antipsychotic medication for at least 1 year before TD assessment. Exclusion criteria included type II diabetes, head injury with loss of consciousness, and seizure disorder. TD occurrence was classified based on the Schooler and Kane criteria using the AIMS (Schooler and Kane, 1982), with individuals having at least one moderate rating or at least two mild ratings on the first seven items in the AIMS being categorized as having probable TD (Schooler and Kane, 1982). Our sample has over $80 \%$ power to detect an effect size (variance explained $\mathrm{Rg}^{2}$ ) of $0.06[\alpha=0.05$, Quanto v1.2.3; (Gauderman and Morrison, 2006)]. In addition, we included an independent sample of 49 participants from the IMPACT (The Individualized Medicine: Pharmacogenetics Assessment and Clinical Treatment) naturalistic pharmacogenetic study. These participants were of self-reported European ancestry who were clinically diagnosed with schizophrenia or schizoaffective disorder and assessed for tardive dyskinesia (Zai et al., 2018c \#574; Herbert, 2018 \#470). Total AIMS scores were available for all 136 patients. We obtained informed consent from each participant enrolled and the study was approved by the CAMH research ethics board.

\section{Genetic Data Collection}

Genomic DNA was extracted from whole blood using high-salt method (Lahiri and Nurnberger, 1991) or extracted from saliva (DNA Genotek) using automated DNA extraction (Chemagen). In the Sekar et al. (2016) paper, the authors used droplet digital PCR (ddPCR) to determine the precise copy numbers of $C 4 \mathrm{~A}$, C4B, C4L, and C4S, with RPP30 as the diploid control. Then, selecting for individuals with at least one copy of $\mathrm{C} 4 \mathrm{~S}$, they amplified the C4S using long-range PCR, and by using ddPCR, determine the number of C4AS and C4BS, and thereafter infer the copy number of C4AL and C4BL. We used a similar approach to determine the number of C4AL, C4BL, C4AS, and C4BS for our study. Copy number assays for the four structural elements [C4A (Hs07226349_cn), C4B (Hs07226350_cn), C4L (Hs07226352_cn), and C4S (Hs07226351_cn)] were run on Viia 7 Real-time PCR System (Thermo Fisher Scientific) in quadruplicates with RNaseP reference assay following manufacturer's protocol, and the copy numbers of $C 4 \mathrm{~A}, C 4 \mathrm{~B}$, C4L, and C4S were determined using the CopyCaller software (Thermo Fisher Scientific). For individuals with at least one copy of C4S, we followed up by running long-range PCR with standard protocol using the following primers specific for $C 4 \mathrm{~S}$ : forward 5'-TCAGCATGTACAGACAGGAATACA-3' and reverse 5'-GAGTGCCACAGTCTCATCATTG-3' (TaKaRa, Clontech) (Sekar et al., 2016). We subsequently ran a TaqManbased genotyping assay to determine the presence of $C 4 \mathrm{~A}$ and/ or $C 4 \mathrm{~B}$ within the $C 4 \mathrm{~S}$ long-range PCR product (Thermo Fisher Scientific). From this follow-up step, we determined the number of C4AS and C4BS in our sample, and then deduced the number of C4AL and C4BL by subtracting from the total numbers of C4A, C4B, C4L, and C4S. Genotype quality was checked by the 
TABLE 1 | Characteristics of the samples analyzed in this study.

\begin{tabular}{lcc}
\hline Characteristics & GR & IMPACT \\
\hline Sex (Male/Female) & $51 / 32$ & $35 / 11$ \\
Age (mean \pm SD) & $42.34 \pm 9.84$ & $40.17 \pm 12.32$ \\
TD occurrence (Yes/No) & $32 / 51$ & $16 / 30$ \\
Total AIMS scores (mean \pm SD)* & $6.01 \pm 6.74$ & $4.11 \pm 4.62$ \\
C4A copy number & $2.07 \pm 0.66$ & $2.02 \pm 0.58$ \\
C4B copy number & $1.88 \pm 0.59$ & $1.85 \pm 0.36$ \\
C4L copy number & $3.02 \pm 0.95$ & $3.17 \pm 0.93$ \\
C4S copy number & $0.93 \pm 0.87$ & $0.70 \pm 0.66$ \\
C4AL copy number & $1.96 \pm 0.65$ & $1.98 \pm 0.54$ \\
C4BL copy number & $1.06 \pm 0.77$ & $1.20 \pm 0.69$ \\
C4AS copy number & $0.11 \pm 0.31$ & $0.04 \pm 0.21$ \\
C4BS copy number & $0.82 \pm 0.83$ & $0.65 \pm 0.64$ \\
C4A expression & $1.19 \pm 0.33$ & $1.19 \pm 0.32$ \\
C4B expression & $1.81 \pm 0.57$ & $1.81 \pm 0.37$
\end{tabular}

* Significant difference between GR and IMPACT samples, with $p=0.014$.

formula $[C 4 \mathrm{~A}+\mathrm{C} 4 \mathrm{~B}=\mathrm{C} 4 \mathrm{~L}+\mathrm{C} 4 \mathrm{~S}]$ and the presence of longrange PCR product in individuals with at least one copy of $C 4 S$. We re-genotyped the samples where the numbers did not match and could not resolve seven samples, which we excluded from the analyses. More information on the two samples is shown in Table 1.

\section{Statistical Analysis}

Sex ratio, average age, average total AIMS scores, and TD occurrence rate did not differ between the two samples $(p>0.05)$. Thus, we conducted statistical analyses on the pooled dataset. TD occurrence was analyzed with sex using Pearson chi-squared test. The rate of TD was not significantly different between males (34.65\%) and females (43.75\%) in the collective sample $(p>0.05)$. AIMS scores were not normally distributed; thus, we performed log transformation. Log-transformed AIMS scores were compared between males and females using Student's $t$ test, where the findings were not significant $(p>0.05)$. However, significant positive correlation was found between AIMS scores and age $(p<0.001)$. Further, average age was compared between TD-positive and TD-negative groups using Student's $t$ test and was significantly higher in the TD-positive group than the TD-negative group $(p<0.001)$. The analyses of TD occurrence with the numbers of structural elements (C4A, C4B, C4L, and C4S) as well as with the numbers of compound structural forms (C4AL, C4AS, C4BL, and $\mathrm{C} 4 \mathrm{BS}$ ) were performed using logistic regression, with age, sex, and sample set included as covariates. The analyses of log-transformed AIMS scores were carried out using linear regression, with age, sex, and sample set as covariates. We used the following formulas provided by Sekar et al. to predict brain expression (composited from expression measures in up to five brain regions from three postmortem brain samples; Sekar et al., 2016) of C4A and C4B: [C4A expression $=(0.47 \mathrm{x}$ $C 4 \mathrm{AL})+(0.47 \times \mathrm{C} 4 \mathrm{AS})+(0.20 \times \mathrm{C} 4 \mathrm{BL})]$ and $[C 4 \mathrm{~B}$ expression $=$ $(1.03 \times C 4 \mathrm{BL})+(0.88 \times \mathrm{C} 4 \mathrm{BS})]$. The predicted expression levels were analyzed with TD occurrence using logistic regression and log-transformed AIMS scores using linear regression.

\section{RESULTS}

The copy number distributions of $C 4$ structural variants in our samples are shown in Figure 1. The copy numbers of structural elements $(C 4 \mathrm{~A}, C 4 \mathrm{~B}, C 4 \mathrm{~L}, C 4 \mathrm{~S})$ and compound structural forms (C4AL, C4AS, and C4BS) were not associated with TD occurrence or TD severity in our pooled sample $(p>0.05)$. We observed a nominally significant association of $C 4 \mathrm{BL}$ with TD severity $(B( \pm \mathrm{SE})=0.118 \pm 0.05 ; t=2.36, p=0.020$; Figure 2$)$. Neither estimated $C 4 \mathrm{~A}$ nor $C 4 \mathrm{~B}$ expression was associated with TD occurrence or TD severity $(p>0.05)$.

\section{DISCUSSION}

This is the first study to examine the $C 4$ gene in TD, and we found a possible association between C4BL copy number and TD severity. However, it is important to note that due to the exploratory nature of our study, we did not correct for multiple testing, and our findings with copy number of C4BL would not have survived Bonferroni correction.

While previous studies have implicated low copy number of C4A (Li et al., 2017; Juptner et al., 2018) in autoimmune diseases, including systematic lupus erythematosus and rheumatoid arthritis, increased $\mathrm{C} 4 \mathrm{~A}$ was associated with schizophrenia susceptibility, possibly through its role in synaptic pruning (Sekar et al., 2016). The role of C4B in autoimmune diseases and schizophrenia needs further studies (Li et al., 2017), but low C4B copy number has been implicated in cardiovascular disease risk (Blasko et al., 2008). While C4A targets amino-group containing antigens, $\mathrm{C} 4 \mathrm{~B}$ preferentially targets carbohydrate antigens (Blanchong et al., 2001). One potential hypothesis for the observed association here is that disrupted sphingolipid metabolism by high-TD-risk antipsychotics such as haloperidol (McClay et al., 2015) may trigger C4B to mediate the white matter disruption observed in TD patients (Bai et al., 2009). Interestingly, a genomewide association study has implicated the proteolipid protein 1 (PLP1) gene in severity of extrapyramidal side effect as measured by the Simpson-Angus Scale (Aberg et al., 2010). Further studies of white matter integrity in TD patients may be warranted.

In addition, complement component $C 3$ has been implicated in metabolic syndrome in schizophrenia patients treated with clozapine (reviewed in Woo et al., 2019) Genes coding for other members in the complement systems that have been associated with schizophrenia susceptibility (Schizophrenia Working Group of the Psychiatric Genomics Consortium, 2014), including the CUB and Sushi multiple domains 1 (CSMD1), C2, and Complement Factor B $(C F B)$, may be an avenue for future genetic investigations of the complement system in TD.

Our study has a number of limitations. In addition to structural elements and compound structural forms examined in this study, the compound structural forms may be configured in various combinations, or structural haplotypes, which will require additional molecular analyses and parental genotypes to resolve. Thus, we were unable to determine the $C 4$ structural haplotypes with our experimental approach and we were not able to resolve the copy number of C4AL, C4BL, C4AS, or C4BS 


\section{A C4 structural elements}
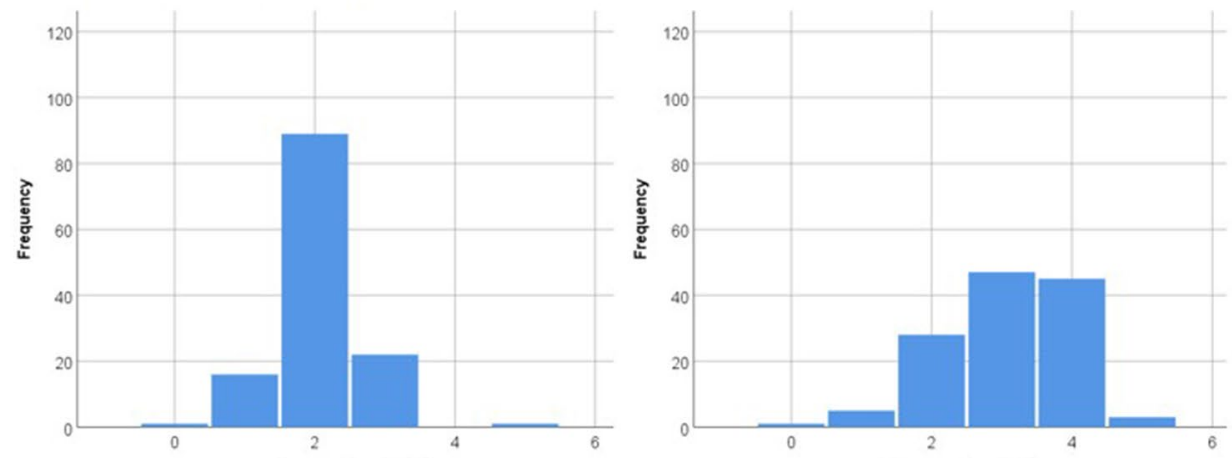

Copy number of C4A
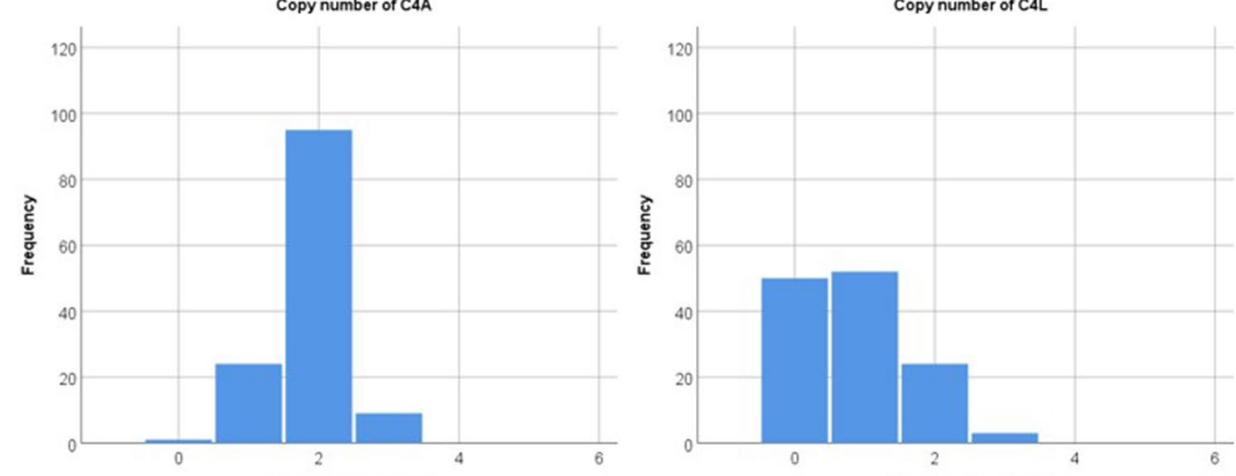

Copy number of $\mathrm{C} 4 \mathrm{~B}$

Copy number of C4S

\section{B C4 compound structural forms}
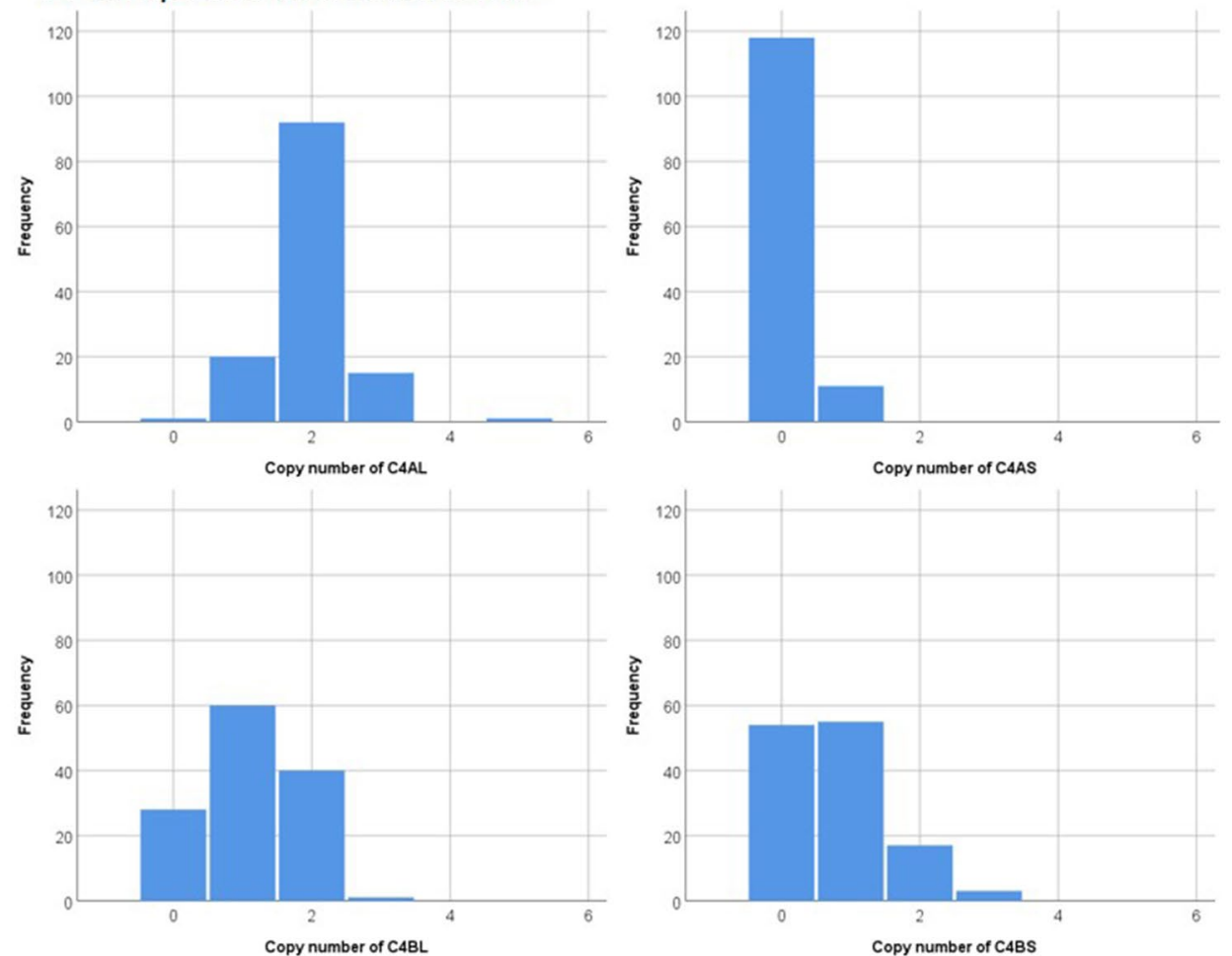

FIGURE 1 | Distributions of copy numbers of the various C4 (A) structural elements and (B) compound structural forms in our study sample. 


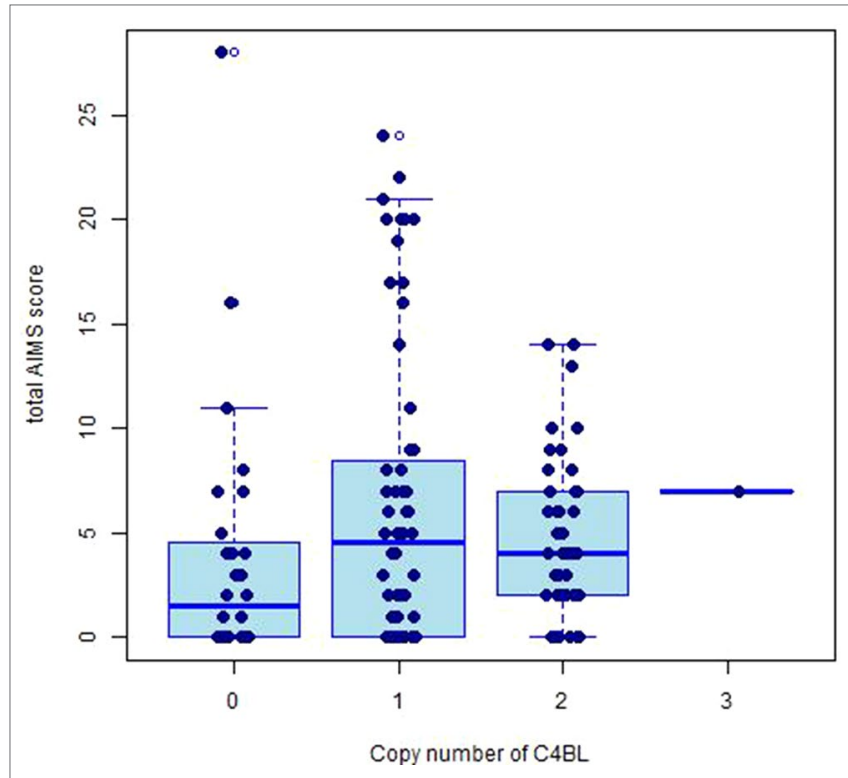

FIGURE 2 | Box plot displaying the distributions of total AIMS scores for copy numbers of $\mathrm{C} 4 \mathrm{BL}$.

in about $1 \%$ of the sample using our experimental workflow. Our sample was of moderate size; thus, while our suggestive findings with $C 4 \mathrm{BL}$ may be due to chance, our lack of significant findings with $\mathrm{C} 4 \mathrm{~A}$ expression and $\mathrm{C} 4 \mathrm{~B}$ expression could be due to insufficient sample size. We also do not have detailed information on clinical variables that could have influenced the findings. These variables include age at onset of TD, treatment duration before TD onset. Thus, further studies in larger samples are required before these findings can be firmly established. Overall, our preliminary findings encourage additional studies of the $C 4$ gene in schizophrenia-related phenotypes including antipsychotic medication response and side effects.

\section{DATA AVAILABILITY STATEMENT}

The datasets for this manuscript are not publicly available because of issues of data ownership and participant consent. Requests to

\section{REFERENCES}

Aberg, K., Adkins, D. E., Bukszar, J., Webb, B. T., Caroff, S. N., Miller, D. D., et al. (2010). Genomewide association study of movement-related adverse antipsychotic effects. Biol. Psychiatry 67, 279-282. doi: 10.1016/j.biopsych.2009.08.036

An, H. M., Tan, Y. L., Shi, J., Wang, Z. R., Soars, J. C., Wu, J. Q., et al. (2015). Altered IL-2, IL-6 and IL-8 serum levels in schizophrenia patients with tardive dyskinesia. Schizophr. Res. 162, 261-268. doi: 10.1016/j.schres.2014.12.037

APA (American Psychiatric Association). (2000). Diagnostic and statistical manual of mental disorders (4th edn - text revision). Washington DC.

Bahia El Idrissi, N., Bosch, S., Ramaglia, V., Aronica, E., Baas, F., and Troost, D. (2016). Complement activation at the motor end-plates in amyotrophic lateral sclerosis. J. Neuroinflammation 13, 72. doi: 10.1186/s12974-016-0538-2

Bai, Y. M., Chou, K. H., Lin, C. P., Chen, I. Y., Li, C. T., Yang, K. C., et al. (2009). White matter abnormalities in schizophrenia patients with tardive dyskinesia: access the datasets should be directed to Dr. James L. Kennedy, jim. kennedy@camh.ca, or Dr. Clement C.Zai, clement.zai@camh.ca.

\section{ETHICS STATEMENT}

The studies involving human participants were reviewed and approved by CAMH Research Ethics Board. The patients/ participants provided their written informed consent to participate in this study.

\section{AUTHOR CONTRIBUTIONS}

$\mathrm{CZ}$ and NF oversaw the overall genetic study design. DH, HE, $\mathrm{SC}, \mathrm{NB}, \mathrm{AV}$, and GR were involved in participant recruitment. SS and MT prepared the DNA samples. CZ, JG, and MT genotyped the samples. CZ and AT conducted the statistical analyses. CZ prepared the first draft of the manuscript. GZ, JP, AT, DM, GR, and $\mathrm{JK}$ reviewed the manuscript.

\section{FUNDING}

CZ, AT, and JK were supported by Genome Canada Genomic Applications Partnership Program (GAPP) and the CAMH Foundation. DM is supported by the Canadian Institutes of Health Research (CIHR Operating Grant MOP 142192), the National Institutes of Health (R01MH085801), the Centre for Addiction and Mental Health Foundation (Joanne Murphy Professorship) and received a Brain \& Behaviour Research (NARSAD) Independent Investigator Award, the Michael Smith New Investigator Salary Prize for Research in Schizophrenia (CIHR) and an Early Researcher Award by the Ministry of Research and Innovation of Ontario. GR is supported by the Canadian Institutes of Health Research (CIHR), as well as the Research Hospital Fund - Canadian Foundation for Innovation (RHF-CFI). We would also like to express gratitude towards Larry and Judy Tanenbaum for their generous support in creating the Tanenbaum Centre for Pharmacogenetics, which is advancing research for the CAMH Pharmacogenetic Program. We would like to thank all the subjects who generously participated in this study. We thank the Ministry of Research and Innovation of Ontario for funding the IMPACT project.

a diffusion tensor image study. Schizophr. Res. 109, 167-181. doi: 10.1016/j. schres.2009.02.003

Blanchong, C. A., Chung, E. K., Rupert, K. L., Yang, Y., Yang, Z., Zhou, B., et al. (2001). Genetic, structural and functional diversities of human complement components $\mathrm{C} 4 \mathrm{~A}$ and $\mathrm{C} 4 \mathrm{~B}$ and their mouse homologues, Slp and C4. Int. Immunopharmacol. 1, 365-392. doi: 10.1016/S1567-5769(01)00019-4

Blaskó, B., Kolka, R., Thorbjornsdottir, P., Sigurdarson, S. T., Sigurdsson, G., Rónai, Z., et al. (2008). Low complement C4B gene copy number predicts short-term mortality after acute myocardial infarction. Int. Immunol. 20 (1), 31-37. doi: 10.1093/intimm/dxm117

Gauderman, W. J., and Morrison, J. M. (2006). "QUANTO 1.1: a computer program for power and sample size calculations for genetic-epidemiology studies", in QUANTO Software. Available at: http://hydra.usc.edu/gxe.

Herbert, D., Neves-Pereira, M., Baidya, R., Cheema, S., Groleau, S., Shahmirian, A., et al. (2018). Genetic testing as a supporting tool in prescribing psychiatric 
medication: design and protocol of the IMPACT study. J. Psychiatr. Res. 96, 265-272. doi: 10.1016/j.jpsychires.2017.09.002

Juptner, M., Flachsbart, F., Caliebe, A., Lieb, W., Schreiber, S., Zeuner, R., et al. (2018). Low copy numbers of complement C4 and homozygous deficiency of C4A may predispose to severe disease and earlier disease onset in patients with systemic lupus erythematosus. Lupus 27, 600-609. doi: $10.1177 / 0961203317735187$

Khandaker, G. M., and Dantzer, R. (2016). Is there a role for immune-to-brain communication in schizophrenia? Psychopharmacol. (Berl.) 233, 1559-1573. doi: 10.1007/s00213-015-3975-1

Lahiri, D. K., and Nurnberger, J. I. Jr. (1991). A rapid non-enzymatic method for the preparation of HMW DNA from blood for RFLP studies. Nucleic Acids Res. 19, 5444. doi: 10.1093/nar/19.19.5444

Lee, H.-J., and Kang, S.-G. (2011). Genetics of tardive dyskinesia. Int. Rev. Neurobiol. 98, 231-264. doi: 10.1016/B978-0-12-381328-2.00010-9

Li, N., Zhang, J., Liao, D., Yang, L., Wang, Y., and Hou, S. (2017). Association between $\mathrm{C} 4, \mathrm{C} 4 \mathrm{~A}$, and $\mathrm{C} 4 \mathrm{~B}$ copy number variations and susceptibility to autoimmune diseases: a meta-analysis. Sci. Rep. 7, 42628. doi: 10.1038/ srep 42628

Liu, H., Kang, Y., Liang, J., Li, C., Xiu, M., Chen, D., et al. (2012). Lower serum interleukin-2 levels in schizophrenic patients with tardive dyskinesia. Psychiatry Res. 198, 329-331. doi: 10.1016/j.psychres.2012.01.002

Mack, M., Bender, K., and Schneider, P. M. (2004). Detection of retroviral antisense transcripts and promoter activity of the HERV-K(C4) insertion in the MHC class III region. Immunogenetics 56 (5), 321-332. doi: 10.1007/ s00251-004-0705-y

McClay, J. L., Vunck, S. A., Batman, A. M., Crowley, J. J., Vann, R. E., Beardsley, P. M., et al. (2015). Neurochemical metabolomics reveals disruption to Sphingolipid metabolism following chronic haloperidol administration. J. Neuroimmune Pharmacol. 10, 425-434. doi: 10.1007/s11481-015-9605-1

Müller, D. J., Schulze, T., Knapp, M., Held, T., Krauss, H., Weber, T., et al. (2001). Familial occurrence of tardive dyskinesia. Acta Psychiatrica Scandinavica 104, 375-379. doi: 10.1034/j.1600-0447.2001.00401.x

Rapaport, M. H., and Lohr, J. B. (1994). Serum-soluble interleukin-2 receptors in neuroleptic-naive schizophrenic subjects and in medicated schizophrenic subjects with and without tardive dyskinesia. Acta Psychiatr. Scand. 90, 311315. doi: 10.1111/j.1600-0447.1994.tb01599.x

Schizophrenia Working Group of the Psychiatric Genomics Consortium. (2014). Biological insights from 108 schizophrenia-associated genetic loci. Nature 511, 421-427. doi: 10.1038/nature13595.

Schooler, N. R., and Kane, J. M. (1982). Research diagnoses for tardive dyskinesia. Arch. Gen. Psychiatry 39, 486-487. doi: 10.1001/archpsyc.1982.04290040080014

Sekar, A., Bialas, A. R., de Rivera, H., Davis, A., Hammond, T. R., Kamitaki, N., et al. (2016). Schizophrenia risk from complex variation of complement component 4. Nature 530, 177-183. doi: 10.1038/nature16549

Shi, J., Levinson, D. F., Duan, J., Sanders, A. R., Zheng, Y., Pe’er, I., et al. (2009). Common variants on chromosome $6 \mathrm{p} 22.1$ are associated with schizophrenia. Nature 460, 753-757. doi: 10.1038/nature08192

Singhrao, S. K., Neal, J. W., Morgan, B. P., and Gasque, P. (1999). Increased complement biosynthesis by microglia and complement activation on neurons in Huntington's disease. Exp. Neurol. 159, 362-376. doi: 10.1006/ exnr.1999.7170

Stefansson, H., Ophoff, R. A., Steinberg, S., Andreassen, O. A., Cichon, S., Rujescu, D., et al. (2009). Common variants conferring risk of schizophrenia. Nature 460, 744-747. doi: 10.1038/nature08186

Sun, H., Wang, F., Fan, H., Yan, Q., Cui, K., Yuan, W., et al. (2013). The interaction of polymorphisms of IL10 and DBH was associated with general symptoms of PANSS with TD in Chinese Han schizophrenic patients. PloS One 8, e70963. doi: 10.1371/journal.pone. 0070963

Tian, L., Tan, Y., Chen, D., Lv, M., Tan, S., Soares, J. C., et al. (2014). Reduced serum TNF alpha level in chronic schizophrenia patients with or without tardive dyskinesia. Prog. Neuropsychopharmacol. Biol. Psychiatry 54, 259-264. doi: 10.1016/j.pnpbp.2014.06.012

van Kesteren, C. F., Gremmels, H., de Witte, L. D., Hol, E. M., Van Gool, A. R., Falkai, P. G., et al. (2017). Immune involvement in the pathogenesis of schizophrenia: a meta-analysis on postmortem brain studies. Transl. Psychiatry 7, e1075. doi: 10.1038/tp.2017.4

Wang, F., Fan, H., Sun, H., Yang, F., Luo, Y., Liu, H., et al. (2012). Association between TNF-alpha promoter -308A/G polymorphism and tardive dyskinesian Chinese Han patients with schizophrenia. Prog. Neuropsychopharmacol. Biol. Psychiatry 37, 106-110. doi: 10.1016/j.pnpbp.2011.12.007

Weinhold, P., Wegner, J. T., and Kane, J. M. (1981). Familial occurrence of tardive dyskinesia. J. Clin. Psychiatry 42, 165-166.

Woo, J. J., Pouget, J. G., Zai, C. C., and Kennedy, J. L. (2019). The complement system in schizophrenia: where are we now and what's next? Mol. Psychiatry doi: 10.1038/s41380-019-0479-0

Wu, Y. L., Savelli, S. L., Yang, Y., Zhou, B., Rovin, B. H., Birmingham, D. J., et al. (2007). Sensitive and specific real-time polymerase chain reaction assays to accurately determine copy number variations (CNVs) of human complement C4A, C4B, C4-long, C4-short, and RCCX modules: elucidation of C4 CNVs in 50 consanguineous subjects with defined HLA genotypes. J. Immunol. 179, 3012-3025. doi: 10.4049/jimmunol.179.5.3012

Yang, Y., Chung, E. K., Zhou, B., Blanchong, C. A., Yu, C. Y., Füst, G., et al. (2003). Diversity in intrinsic strengths of the human complement system: serum C4 protein concentrations correlate with $\mathrm{C} 4$ gene size and polygenic variations, hemolytic activities, and body mass index. J. Immunol. 171 (5), 2734-2745.

Yassa, R., and Ananth, J. (1981). Familial tardive dyskinesia. Am. J. Psychiatry 138, 1618-1619. doi: 10.1176/ajp.138.12.1618

You, Z., Yang, J., Takahashi, K., Yager, P. H., Kim, H. H., Qin, T., et al. (2007), Reduced tissue damage and improved recovery of motor function after traumatic brain injury in mice deficient in complement component C4. J. Cereb. Blood Flow Metab. 27, 1954-1964. doi: 10.1038/sj.jcbfm.9600497

Zai, C. C., Tiwari, A. K., Zai, G. C., Maes, M. S., and Kennedy, J. L. (2018a). New findings in pharmacogenetics of schizophrenia. Curr. Opin. Psychiatry 31, 200212. doi: 10.1097/YCO.0000000000000417

Zai, C. C., Maes, M. S., Tiwari, A. K., Zai, G. C., Remington, G., and Kennedy, J. L. (2018b). Genetics of tardive dyskinesia: promising leads and ways forward. J. Neurol. Sci. 389, 28-34. doi: 10.1016/j.jns.2018.02.011

Zai, C. C., Lee, F. H., Tiwari, A. K., Lu, J. Y., de Luca, V., Maes, M. S., et al. (2018c). Investigation of the HSPG2 gene in tardive dyskinesia - new data and metaanalysis. Front. Pharmacol. 9, 974. doi: 10.3389/fphar.2018.00974

Zai, C. C., Hwang, R. W., De Luca, V., Muller, D. J., King, N., Zai, G. C., et al. (2007). Association study of tardive dyskinesia and twelve DRD2 polymorphisms in schizophrenia patients. Int. J. Neuropsychopharmacol. 10, 639-651. doi: $10.1017 /$ S1461145706007152

Zai, C. C., Tiwari, A. K., Mazzoco, M., De Luca, V., Muller, D. J., Shaikh, S. A., et al. (2013). Association study of the vesicular monoamine transporter gene SLC18A2 with tardive dyskinesia. J. Psychiatr. Res. 47, 1760-1765. doi: 10.1016/j.jpsychires.2013.07.025

Conflict of Interest: The authors declare that the research was conducted in the absence of any commercial or financial relationships that could be construed as a potential conflict of interest.

Copyright $\odot 2019$ Zai, Tiwari, Zai, Freeman, Pouget, Greco, Tampakeras, Shaikh, Herbert, Emmerson, Cheema, Braganza, Müller, Voineskos, Remington and Kennedy. This is an open-access article distributed under the terms of the Creative Commons Attribution License (CC BY). The use, distribution or reproduction in other forums is permitted, provided the original author(s) and the copyright owner(s) are credited and that the original publication in this journal is cited, in accordance with accepted academic practice. No use, distribution or reproduction is permitted which does not comply with these terms. 